\title{
High Reliability Care: At the Intersection of Policy and Practice
}

\author{
Mary K. Walker ${ }^{1 \star}, \mathrm{PhD}, \mathrm{RN}$, FAAN and Thomas C. Walker ${ }^{2}, \mathrm{PhD}$
}

${ }^{1}$ Director and Professor, University of Maine, School of Nursing, US

${ }^{2}$ Postdoctoral Fellow, South Carolina Water Resources Center, Clemson University, USA
*Corresponding author

Mary K. Walker, PhD, RN, FAAN, Director and Professor, University of Maine, School of Nursing, 5724 Dunn Hall, Room 201, Orono, ME 044695724, E-Mail: mary.walker1@maine.edu

Submitted: 29 June 2018; Accepted: 10 July 2018; Published: 18 July 2018

\begin{abstract}
Health care organizations in the United States struggle to maintain safety and provide quality patient care. In a complex policy environment, the Joint Commission has directed its efforts toward helping health systems achieve high reliability health care. Heath care organizations, facing both accreditation imperatives and political challenges, are mired in the uncertainty of resource availability. The challenges of high reliability in a high stakes industry elude even the most seasoned CEOs and administrators. In particular, it is essential at this time is to pinpoint how public health policy, when coupled with development of high reliability culture, informs implementation of quality and safety at the local level and advances Joint Commission directives related to high reliability care. This theoretically focused paper explores the phenomena of quality and safety from the vantage of two differing lenses, practice and policy. The theoretical analysis of high reliability health care (policy, organizational structure, and actors) contributes to further understanding the challenges facing high reliability patient care implementation throughout hospital systems in the United States. Discussion highlights appropriateness of model fit, whether a top down approach to patient care is realistic, and possible challenges of a centralized policy in an inherently decentralized industry environment. Conclusions reinforce the need for local health care systems and administrators to adopt and adapt the Joint Commission's high reliability model to their system to correct industry failures.
\end{abstract}

Keywords: health services administration, capacity building, organizational policy, delivery of health care

\section{Introduction}

Health care quality is a complex phenomenon. Concepts such as quality of care, clinical translational science, patient safety, best practices, health outcomes and other placeholders populate the quality landscape. Health care quality is intended to convey that individuals, families, communities and populations can expect that, when care is required, care will be delivered consistent with contemporary science and best efforts to uphold the highest standards of clinical practice.

There is general consensus that patient safety is the first indicator or building block of health care quality. The Institute of Medicine (IOM) [1] defined health care quality as "the degree to which health care services for individuals and populations increase the likelihood of desired health outcomes and are consistent with current professional knowledge" [2]. Despite a well-accepted definition, quality and safety efforts proved far more difficult to measure and improve than to define. Efforts over the last twenty years advanced systems of care delivery that (1) prevented errors; (2) learned from the errors that occurred; and (3) were built on a culture of safety that involved health care professionals, organizations, and patients. Quality dashboards became a staple in evaluation and Trustee circles, lending credence to health care quality as a crucial variable in health care delivery as well as health outcomes research. Despite rapid adoption, health care organizations (HCOs) were left to their own devices with respect to defining, implementing, measuring and evaluating what constituted health care quality. Thus, changes that appeared to impact safety and care quality in one organization were not readily transferable to other organizations. To that end, the Institute for Healthcare Improvement and other organizations attempted to enroll HCOs in collective efforts to improve safety, defined for measurement purposes as "days" that were error-free in a series of domains (ventilator-acquired pneumonia, e.g.).

Despite these efforts, HCOs continued to be presented with data that showed otherwise. Patrick Conway, Chief Medical Officer of the Centers for Medicare \& Medicaid Services (CMS) (2013) [3], for example, cited persistent concerns regarding safety and quality outcomes in the nation's hospitals and health science centers. Conway (2013) noted that 1 of every 8 patients nationally suffered injury or a potentially avoidable complication during a hospital stay [4]. Harvard researchers found no evidence that changes implemented by most acute care facilities led to lower infection rates, underscoring the care complexities facing hospitals $[5,6]$.

With persistent lack of uniform progress at the local level, the Joint Commission (TJC) took a top-down approach to raising quality 
standards in US hospitals. TJC adopted a high reliability care policy to take corrective action to address industry failure. With this research, the objective is to pinpoint how public health policy, when coupled with development of high reliability culture, informs implementation of quality and safety at the local level and advances TJC directives related to high reliability care.

\section{Scope of the Problem}

Following sharply on the heels of Conway's assertions, Medicareimplemented payment reforms [7] included withholding reimbursement for additional costs resulting from patient treatment for avoidable complications: readmission within 30 days within six specific categories of treatment or too early discharge. 761 facilities, equaling $1 / 4$ of the nation's hospitals, were identified as targets for payment reform citation resulting from persistent safety and quality concerns [3]. While some improvements were noted, CMS still withheld $\$ 528 \mathrm{M}$ from more than $2500 \mathrm{HCOs}$ as a result of 30 day readmission variances in FY 2017 [8]. Those health systems with the worst rates of treatment-related injury lost $1 \%$ of Medicare payments for a year. Conway tersely summed up the current climate: "We want hospitals focused on patient safety and we want them laser-focused on eliminating patient harm" [4].

HCOs grappled with practice variability by placing consistent attention on what not to do, for example, not touching patients without engaging in prior handwashing. Emphasizing what not to do, however, rarely assures that health care providers will know, intuitively, the correct course of action to take in any given situation or support it. For example, health-care providers practice hand hygiene less than half as much as they should, according to the U.S. Centers for Disease Control and Prevention [9]. In fact, handwashing is one of the most difficult benchmarks on which to show improvement. Henry Ford Hospital addressed this issue through a series of quality studies that demonstrated conclusively that bacteria within their own facilities could be cultured from unused gloves, the computer mouse at a nurses' station, medication carts, etc. The intervention to improve handwashing included creating a culture of "disgust" rather than inattention at the notion of bacterial contamination [10]. Data indicated that, following circulation of materials developed expressly for the purpose, handwashing on four targeted low-performing units improved by as much as $50 \%$. Thus, consensus regarding health care quality and safety acknowledges that knowing what to do and when to do it is an emerging field of science and policy [11].

The handwashing data underscore that the simple acknowledgement that persistent variations in outcomes appear to defy explanation is a good place to start. Failure to achieve quality outcomes might be attributed to shortfalls in: (1) talent; (2) time; or (3) treasure, meaning that clinicians are not skilled enough, resources are not plentiful enough, or allotted hospitalization time is not sufficient to achieve the best clinical outcomes for all persons [12]. Hospitals find improvement difficult to sustain, reporting "project fatigue" because so many problems need attention [11]. Further, no hospitals or health systems demonstrate excellence throughout their institutions, supporting the dictum that complex systems have more points of failure [13].

Despite IOM dictums $(2001,2003)[14,15]$ regarding the hard work of improving health care quality, Heifetz (2006) noted that most organizations, left to their own devices, nibble around the edges of problems, throwing one well-known technical solution after another at persistent challenges, rather than attempting to identify, understand and tackle the thorny issues underlying, creating or contributing to organizational problems [16]. While technical solutions are familiar and can be implemented quickly, they are inadequate when applied to intractable problems within organizations. The single greatest challenge for health care systems now is accurate categorization of problems as either adaptive or technical. Those that are technical are readily resolved with information at hand. Those that are adaptive in nature require fundamental organizational change to affect positive and sustained outcomes. For example, the simple addition of greater numbers of Registered Nurses (RN) without regard to clinical assignment or educational qualifications may not solve problems of surgical patient mortality, as evidenced in the following investigation. Aiken et al, 2014 addressed the increasingly common practice of RN staffing austerity [17]. These investigators obtained discharge data for 422,730 surgical patients from 300 hospitals in nine European countries. The patients were 50 years of age and older. The investigators also distributed surveys to 26,516 practicing nurses in study hospitals. The surveys were used to assess both nurse staffing and nurse education. Investigators used generalized estimating equations to assess the effects of nursing factors on the likelihood of surgical patients dying within 30 days of admission, before and after adjusting for other hospital and patient characteristics.

An increase in nurses' workload by one patient increased the likelihood of an inpatient dying within 30 days of admission by $7 \%$ (odds ratio $1 \cdot 068,95 \%$ CI $1 \cdot 031-1 \cdot 106$ ). On the other hand, every $10 \%$ increase in bachelor's degree prepared nurses was associated with a decrease in this likelihood by $7 \%(0.929,0 \cdot 886-0.973)$. The data imply that patients in hospitals in which $60 \%$ of nurses held bachelor's degrees and where nurses cared for an average of six patients would have almost $30 \%$ lower mortality than patients in hospitals in which only $30 \%$ of nurses had bachelor's degrees and nurses cared for an average of eight patients. The data argue for strict controls on two variables in order to reduce post-surgical mortality within the 30 day window: numbers of post-surgical patients assigned to each staff RN and BSN preparation of a large preponderance of nursing staff, consistent with IOM (2010) recommendations [18]. These data corroborated earlier findings of Aiken (2003) and her investigative team using Medicare data reported by United States HCOs to the CMS [19].

\section{High Reliability Care}

TJC devised a strategy to advance high reliability in HCOs by asserting that member organizations systematically engage in high reliability efforts or face loss of accreditation. High-reliability science is the study of high stakes organizations, such as commercial aviation and nuclear power industries, that operate under hazardous conditions. Adapting and applying the lessons of high stakes organizations to healthcare offer hospitals important opportunities to reach levels of quality and safety that are comparable to those of the best high stakes organizations.

TJC's article on high reliability health care [20] and the recommended framework for application by hospital systems supports systematic change in both organizational and provider behavior related to patient safety. The framework that TJC developed is a significant step forward in directing the evolution toward improved patient outcomes in the healthcare industry as a whole. Yet, HCO efforts 
in health care remain to be applied in a way that meaningfully meet TJC's standards of high reliability. There are several possible reasons for stagnation of high reliability initiatives. Contrary to the efforts of HCOs to engage in a series of programs and projects to address individual quality challenges, TJC holds that addressing deep change begins with creating and sustaining high reliability organizations.

TJC standards serve to move HCOs towards a unified standard of health care delivery nationwide, in contrast to the emphasis on individual efforts that characterized quality and safety activities a decade ago. As is the case with most policy areas and consistent with political culture, some hospitals have already exceeded TJC's minimal requirements of accreditation. In acknowledgement of $\mathrm{HCO}$ efforts to attain high reliability goals, many organizations earned and maintain The Joint Commission's Gold Seal of Approval TM, a nationwide symbol of quality reflecting organizations' commitment to quality [21]. Yet, despite the best ongoing efforts of these systems, high reliability has yet to be attained by any health system in the United States.

TJC asserts that high reliability standards and their outcomes will not likely come through regulation alone. Rather, given that all hospital politics are "local", the likely mechanism for lasting change includes both administrative and practitioner champions and "early adopters": hospital boards, directors, CEOs, as well as patients, doctors, nurses, and staff of individual hospitals. The recommendation supports that HCOs assume responsibility for the TJC imperative, consistent with strategies of adaptive change. TJC continues to offer its expertise, along with its framework, in order to help health systems move in the direction of high reliability health care.

Both scale and implementation issues stymy timely adoption of TJC's imperative. An important adaptive step in moving policy into practice relates to modeling both processes and outcomes prior to implementation. Such virtual modeling assures organizations the ability to pinpoint organizational, staff, and patient-centered elements that are sensitive to intervention [22].

Effken and colleagues (2009), showed that task complexity, staffing and environmental turbulence varied on a unit-by-unit basis within the same organization [22]. The impact of these variables was constant; the differential effect of variables on quality and safety varied by unit. Thus, the unique culture of units required unit- specific innovation to achieve quality and safety outcomes throughout the organization. Virtual modeling prior to implementation identified strategies that improved virtual performance. Virtual modeling, therefore, may represent an important theoretical step in assuring positive and consistent real world outcomes in both quality and safety. Modeling, then, maximizes resource utilization, effectively saving organizations both time and money in their innovative high reliability efforts.

Vogus and Iacobucci (2016) examined one such adaptive approach to emulating high-reliability organizations (e.g., nuclear power plants) [23]. The approach invoked a combination of specific work practices and behavioral processes to detect and adapt to unexpected events in order to operate in a nearly error-free manner. The investigation addressed whether and how reliability-enhancing work practices (REWPs) helped enable such processes and improve performance (i.e., reduce errors). Using survey and archival data from 1,685 RNs and 95 nurse managers in 95 hospital nursing care units, the authors examine how REWPs affected a set of attitudinal (affective commitment and organizational citizenship behavior) and discursive (respectful interaction and mindful organizing) processes and, in turn, patient safety. The investigators identified that the greater use of REWPs was directly and indirectly (through respectful interaction and mindful organizing) associated with fewer medication errors and patient falls. In contrast, organizational citizenship behavior was associated with higher rates of medication errors and patient falls. These data indicate yet another variable essential to creating and sustaining an error-free healthcare workplace: personal commitment on the part of every person at every level of an organization.

A High Reliability Organization (HRO), therefore, models an environment of "collective mindfulness" wherein all physicians, caregivers, employees, management, and key stakeholders look for, and report, small problems or unsafe conditions before they pose a substantial risk to their patients and when they are easy and affordable to fix. Contemporary literature reveals that implementation of HRO model in clinics and physician-based practices creates improvement in the following outcomes: organizational effectiveness, organizational efficiency, customer satisfaction, compliance, documentation, and organizational culture [21]. Implementing an effective HRO model helps physician practices' leaders and practitioners design their organizations around anticipating and preventing problems [24]. Moving forward, the challenge is to coalesce best practices into substantive policies that serve to guide ongoing implementation of high reliability initiatives and outcomes evaluation. Given the data, it is past time for providers and organizations to address practice variability by improving the interface between data, practice and health policy.

\section{Health Policy Literature}

Policy theory is useful in explaining the process by which policy decision-making takes place. Several contributions have previously introduced some policy frameworks to understanding health policy processes. Hewison (2008) identified gaps in the health policy literature from a nursing perspective [25]. Despite the gaps, health policy literature is growing. The works cited are examples of policy contributions that can provide traction in interpreting high reliability health care and set the stage for the theoretical contribution.

Some investigators support Anderson's (2015) policy stages as useful for guiding their research [26]. While these same investigators have rightfully acknowledged the limitations with such a selection, Anderson's model is still considered useful in guiding health policy understandings in more traditional policy settings [27]. High reliability efforts are currently focused within the policy implementation (high reliability care application in the local hospital setting) stage after proceeding through the problem identification, agenda setting, formulation, and adoption stages. The caveat is that TJC operates outside of the traditional framework for policy-making but can be contextualized using the policy process framework [26] to highlight better understanding of the process.

Malone (2005) considered additional health policy research in an effort to assess the policy environment itself [28]. Similarly, this research highlights policy environment concerns with TJC's high reliability policy environment for successful implementation after agenda setting, formulation, and adoption stages of this policy. It bears repeating that TJC operates outside traditional policy processes; despite this, the policy environment and the interactions between 
the decision-makers and those impacted at the local level cannot be ignored for successful implementation of high reliability objectives.

TJC, in its decision to move toward high reliability health care, reviewed and incorporated years of data and research to support and advance safety and quality outcomes. In this sense, TJC utilized policy-oriented learning, an important component of policy choice wherein TJC, rather than maintaining the status quo and doing nothing, made a decision to move in the direction it did [29].

\section{Organizational Policy Theory}

The theoretical focus of this paper is in the implementation of TJC's high reliability model at the individual hospital level. The policy, having been adopted by TJC, must now be engrained into all hospitals in order to meet the new standard. The theoretical approach of this paper highlights the policy relationship between TJC and the health care organizations that TJC accredits with an understanding of the broader policy environment. The relationship between TJC and its accredited hospitals varies depending on the lens used to operationalize the TJC initiative. A policy lens, briefly reviewed in the policy literature, is appropriate in discussing policy processes; however, as the policy has been adopted, the most appropriate lens is an organization lens to understand how the policy must be implemented.

TJC set new expectations for future accreditation, stipulating compliance with high reliability modelling as a necessary precondition for continued accreditation. The stipulation was softened somewhat by TJC's identification and testing of models and instruments on which to scaffold high reliability initiatives. Further, TJC offered initial direct support, as well as access to instruments and models, supporting systematic assessment of unique internal reliability issues by member organizations themselves.

Applying a principal-agent [30] interpretation of the organizational policy environment (including a centralizing policy objective implemented in a decentralized manner), the health system (agent), is the policy actor required to adopt TJC's policy objective by moving toward high reliability. The principal (TJC), in its formulation of a policy decision regarding high reliability health care, requires policy adoption to match policy implementation. In tying accreditation to implementation, TJC is attempting to assure implementation through an organizational carrot or stick approach.

The stick in this case is the statement that if health systems do not move toward high reliability modelling as framed by TJC, the potential for future accreditation is in jeopardy. This is an interesting approach; although TJC is the recognized accrediting body in the health care industry it holds no specific regulatory power granted by law. Accreditation is and continues to be elective. The question thus remains: How can the principal, TJC, incentivize the agent, accredited member organizations, to adopt the proposed high reliability framework? Using the carrot as incentive, TJC offered services as onsite advisor supporting organizational and early adopter efforts. Using its advisory/mentoring role, TJC provided its members both organizational assessment and planned improvement assistance to strengthen organizational reliability. The organization itself must then engage in its own adaptive change efforts. Heifetz (1994, 2006) outlines 5 strategic principles of leadership consistent with the current need to assure safety and enhance quality in the health care system through high reliability $[16,31]$.
A potential flaw in this model is that the directive overlooks the organizational policy environment of health care. There are many forces at work which may influence a local health system. Expanding the example of principal agent theory, there are many other principal agent relationships within the health policy environment that impact full adoption and implementation of TJC model. These include possible direct Agent-to-Agent interactions with the same Principal responsible for different policy implementation interpretations. A simplified diagram helps convey the complex nature of a centralizing policy objective in an inherently decentralized industry (Figure 1). Whether the system is Federal, State, Local, or any other type, it is considered local in the context of this theoretical application even though there are inherent nuances within that understanding.

- $\quad$ TJC is the accrediting body of health systems but is responsive to the federal environment of this country, both in acknowledging and in complying with federal law and policy. TJC (Principal) also directly impacts hospital boards, their trustees and directors (Agent), integral players to changing organizational culture to meet policy objectives.

- The Board in each hospital (Principal) relates through organizational policy to other Agents, namely the staff who will implement the tenets of high reliability care. The actions of the Board determine if and when professional and paraprofessional staff will make a shift toward high reliability. Then, according to TJC's article (Chassin and Loeb, 2013) the agents bear responsibility for changing the culture through their own incentive structures and imperatives [20].

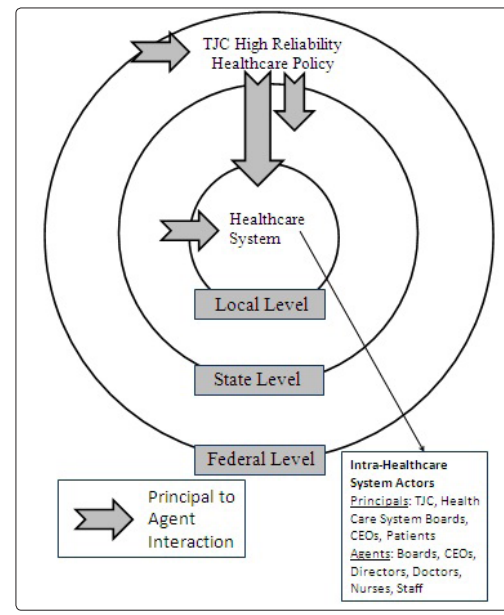

Walker and Walker, 2017

Figure 1: Principal Agent Organizational Policy Environment Diagram

- $\quad$ The patient (Principal) and the doctors, nurses, and staff(Agent) maintain a Principal-Agent relationship in that the Principal (patient) does not wish to suffer harm due to therapeutic misadventure. Federal and State regulatory and enforcement agencies (Principal) impact policy implementation through their interaction and demands for compliance with the health systems (Agent). TJC (Principal) and state agency (Agent) represent a Principal-Agent relationship each time accreditation practices exert influence on state regulators. Since TJC accredits health care organizations, including hospitals, there could be a direct conflict between Agents (state and hospital), each with their own interpretation of policy objectives. 
Multiple organizational alliances could potentially cause a direct implementation conflict at the hospital/local level as the hospital must act out what both TJC and state regulatory bodies require of them. Every state agency could potentially be interpreting both policy and laws differently. Differences in interpretation would make TJC's task more complex and contribute to organizational variation in high reliability implementation. Fundamental organizational theory components must also be addressed. While individuals may or may not be open to change or new ideas related to how best to practice healthcare, organizations vary significantly.

Never has the distinction between "haves" and "have nots" been more evident than in the landscape of TJC's proposal related to high reliability. If quality efforts are the unique purview of the organization itself, paid for from the profits of the institution, outcome variability must be accepted as part of doing business. Yet, as demonstrated in other high stakes industries, the absence of high reliability sets the occasions for spiraling costs associated with morbidity, mortality and legal action, costs borne by the institution itself, in addition to the cost of its quality efforts. Because of these emerging high reliability realities, a number of HCOs dropped their accreditation with the TJC, preferring accreditors that do not advance high reliability requirements.

At the end of the day, once organizations with both the resources and inclination to perform trial and error within their systems have done so, nothing is left for other organizations but to follow suit to remain competitive. Those that lead the way in healthcare organization innovation and those who follow it in order to competitively match what others have done are in line with what could be considered health organization diffusion. Diffusion modeling offers some insight into the "natural progression" of best practices in decentralized organizational policy environments and could inform adoption and implementation of high reliability practices in local health systems. When the model is in place and the bar has been raised, others have little option but to mirror the strategies of competitors contributing to innovative practice spread. Importantly, this leaves the costs of innovation in the hands of relatively few top-performing organizations. Thus, their influence in the health care arena is farreaching and often unchallenged.

TJC high reliability could be fostered quickly in health systems that bring in outside ideas and represent an open model learning organization. An adaptive health system is engaged in looking outward for best practices and is potentially more likely to adopt TJC's recommendations. However, if the health system operates as a closed system, it is more likely to assume that it has the professionals and body of knowledge at its disposal to make the proper organizational alignments internally.

\section{Discussion and Conclusion}

Having introduced theoretical concepts to contextualize TJC's model in the complex organizational policy environment, there are several critical questions that remain to be addressed as TJC rolls out and enforces an agenda of high reliability for health care. These discussion points could help identify further research opportunities moving forward if high reliability care continues to stagnate. There are inevitably some considerations not presented in this discussion which could be areas of future research in high reliability care. There are also limitations to discussions and conclusions based solely on literature review and theoretical non-empirical research.
Is such a unilateral directive on the part of TJC the appropriate way to affect an industry standard? The health care industry, along with the airline and nuclear industries, has the potential for minor errors to manifest as significantly devastating outcomes. It is appropriate to ask whether high reliability thinking and models generated in other high stakes industries can be applied to health care delivery systems, processes and outcomes It remains to be seen whether the reliability efforts of other high stakes agencies and organizations can be used to improve reliability efforts in healthcare systems. TJC suggests that individual health systems and the actors within these systems will ultimately be the driving force in moving the industry toward a standard of high reliability. TJC, while applauding their individual/systems efforts, designed a framework geared toward moving all accredited systems toward a unified standard of high reliability. Realistically, TJC acknowledged that individual health system culture(s) will need to be modified before a high reliability model can be applied at the local level. Despite the assertion, TJC is promulgating a singular model for moving toward this objective.

With individual industry leaders developing their own approaches toward high reliability, is a standardized high reliability model useful or even appropriate? Would it be better to allow industry leaders to continue to develop their own individual frameworks tailored to their own political health culture, consistent with outcomes reported by Effken and colleagues (2009)? Supporting an environment that allows industry leaders to naturally navigate the waters of high reliability suggests that the playing field is level among organizations. The federal government's decision to call back $\$ 750 \mathrm{M}$ in Medicare dollars from industry payers and organizations engaged in quality efforts destabilized the field. This decision created systems of inequality by asserting that quality improvement was an individual organizational affair, funded by the organizations themselves. This unilateral action begs the question of the relationship between quality efforts of the organization and the reliability of the care delivered, care that is funded increasingly through federal, state and private mechanisms.

Is TJC's high reliability proposal a responsible and natural outgrowth of the safety and quality landscape as it currently exists? The actions of TJC in moving toward universal high reliability standards is disruptive by design of the natural progression of health system delivery improvement. Indeed, the proposal forces safety and quality standards toward high reliability. Certainly it is too soon to engage in either quantitative analysis or policy analysis to determine whether TJC's efforts will have a lasting impact on the industry. If TJC is credibly committed to this objective and its framework to meet this objective, it will still need to allow time for the recommendations to take root in the industry. Given political turbulence, policy implementation may be restricted. The concept of "taking root" results from sustained credible commitment. TJC has committed its efforts to high reliability health outcomes and must remain consistent in its directive.

Did TJC, when designing its framework, consult with health systems (other than the identified partners in the projects) and garner stakeholder feedback with their framework before publishing their intended policy objectives? Developing an engagement piece during the policy formulation portion of the policy process, supportive of HCOs to achieve buy-in at the local health system level, is a potentially missed policy piece of achieving 
compliance with the policy. While certain health systems were brought into the decision-making process, attention to receiving feedback from a broader set of health systems might have been prudent before making a final decision. Unless stakeholders are consulted throughout the process and TJC achieves buy-in from the health systems, framework and policy objectives could fail. Within the individual health system, the Board will need to consult its own set of stakeholders to achieve buy-in that allows the model to work and to tailor implementation of the framework/high reliability objective to its culture. The local process itself could take considerable time and resources to achieve buy-in, let alone the time needed for implementing high reliability once buy-in has presumably been achieved.

TJC (2013) offered its expertise, along with its framework, in order to help health systems achieve the reality of high reliability health care. This article addressed both the challenges and opportunities embedded in TJC proposal from both practice and policy perspectives. Potential implementation issues emerging from principal agent theory used to scaffold proposed TJC directives were detailed. Turbulence in the industry, federal and state governments, and individual organizations themselves impact the "will" to design an industry of high reliability and zero harm. In the end, unless thousands of individual health systems in the United States make strategic decisions to move toward high reliability, it remains both an elusive and practical impossibility.

Hospital systems and nursing executives should act within a context of expectations that implementing high reliability care will need to match what was mandated by the initial policy decision. In the case of high reliability care, there is little room for variance from the policy decision. High reliability policy shapes implementation outcomes in that it defines the arena in which the process takes place and the identity and role of those tasked with the organizational change necessary to meet the new standard. What it does not do, by way of local health system culture, is provide a clear path that all systems could take to implement this standard [32, 33].

\section{References}

1. Chassin MR \& Galvin RW (1998) The urgent need to improve health care quality. Institute of Medicine National Roundtable on Health Care Quality. Journal of the American Medical Association 280: 1000-1005. doi:10.1001/jama.280.11.1000

2. Lohr KN (1990) Medicare: A Strategy for Quality Assurance. Washington, DC: National Academy Press.

3. Centers for Medicare and Medicaid Services (2013) CMS Quality Strategy. 2013 - Beyond.Internet, http://www.cms.gov/ Medicare/Quality-Initiatives-Patient-Assessment Instruments/ QualityInitiativesGenInfo/Downloads/CMS-Quality-Strategy. pdf. published November 18, 2013.

4. Conway P (2013) Value-based payment and health system transformation. National Health Policy Forum, November 22, 2013.

5. Institute of Medicine (2011) Healthcare System Complexities, Impediments, and Failures. Engineering a Learning Healthcare System: A Look at the Future: Workshop Summary. Institute of Medicine (US) and National Academy of Engineering (US) Roundtable on Value \& Science-Driven Health Care, Washington, DC: National Academies Press.

6. Zimlichman E, Henderson D, Tamir O, Franz C, Song P, et al. (2013) Health Care-Associated Infections: A Meta- analysis of Costs and Financial Impact on the US Health Care System. Journal of the American Medical Association Internal Medicine 173: 2039-2046. doi: 10.1001/jamainternmed.2013.9763.

7. Centers for Medicare and Medicaid Services (2014) Medicare's delivery system reform initiatives achieve significant savings and quality improvements - off to a strong start. Internet.

8. Rau J (2017) Under Trump, Hospitals face same penalties embraced by Obama. Kaiser Health News, August 3, 2017.

9. Center for Disease Control (2017) Handwashing Saves Lives. https:/www.cdc.gov/handwashing/index.html

10. CNN (2016) This is what made doctors want to wash their hands. Internet, https://www.cnn.com/2016/06/09/health/healthcare-doctors-hand-washing-photo- study/index.html

11. Chassin MR (2017) "Sentinel Event Alert Issue 57: "Building Your Safety Culture: A Job for Leaders”. Webinar Replay. The Joint Commission.

12. Tearte JM (2013) Encore Leadership: Transforming Time, Talent and Treasure into a Legacy that Matters. Crystal Stairs, Inc. Atlanta, GA

13. Cook RI (2000) How Complex Systems Fail. (Being a Short Treatise on the Nature of Failure; How Failure is Evaluated; How Failure is Attributed to Proximate Cause; and the Resulting New Understanding of Patient Safety). Cognitive Technologies Laboratory, University of Chicago.

14. Institute of Medicine (2001) Crossing the Quality Chasm: A New Health System for the 21st Century. Washington, D.C: National Academy Press.

15. Institute of Medicine (2003) Health Professions Education: A Bridge to Quality. National Academies of Science.

16. Heifetz RA (2006) Anchoring Leadership in the Work of Adaptive Progress. The Leader of the Future 2: Visions, Strategies, and Practices for the New Era. Eds. Frances Hesselbein, Marshall Goldsmith. Jossey-Bass.

17. Aiken LH \& RN4CAST consortium (2014) Nurse staffing and education and hospital mortality in nine European countries: a retrospective observational study. The Lancet 383: 1824-30. doi: 10.1016/S0140-6736(13)62631-8

18. Institute of Medicine (2010) The Future of Nursing: Leading Change, Advancing Health. National Academies of Science.

19. Aiken LH, Clarke SP, Cheung RB, Sloane DM, et al. (2003) Educational levels of hospital nurses and surgical patient mortality. Journal of the American Medical Association 290: 1617-1623. doi: 10.1001/jama.290.12.1617

20. Chassin MR \& Loeb JM (2013) High reliability health care: Getting there from here. The Milbank Quarterly 91: 459-490. doi: 10.1111/1468-0009.12023

21. Griffith JR (2015) Understanding high-reliability organizations: Are Baldrige recipients models? Journal of Healthcare Management 60: 44-61.

22. Effken J (2009) Computational modeling: From virtual to actual. Nursing Management 40: 31-34. doi: 10.1097/01. NUMA.0000347411.73201.02

23. Vogus TJ \& Iacobucci D (2016) Creating Highly Reliable Health Care: How Reliability- Enhancing Work Practices Affect Patient Safety in Hospitals. ILR Review 69: 911-938. doi: $10.1177 / 0019793916642759$.

24. Colla CH (2014) Swimming against the current - What might work to reduce low-value care? The New England Journal of Medicine 371: 1280-1283. doi: 10.1056/NEJMp1404503

25. Hewison A (2008) Evidence-based policy: Implications for Nursing and Policy Involvement. Policy, Politics, and Nursing 
Practice 9: 288-298. doi: 10.1177/1527154408323242.

26. Anderson JE (2015) Public policymaking: An introduction. Stamford, CT: Cengage Learning.

27. Ellenbecker CH \& Edward J (2016) Conducting Nursing Research to Advance and Inform Health Policy. Policy, Politics, and Nursing Practice 17: 208-217. doi:10.1177/1527154417700634

28. Malone RE (2005) Assessing the Policy Environment. Policy, Politics, and Nursing Practice 6: 135-143. doi: $10.1177 / 1527154405276141$

29. Jennings CP (2004) The Art and Science of Policy Choice. Policy, Politics, and Nursing Practice 5: 228-232. doi: $10.1177 / 1527154404269377$

30. Jenkins-Smith H, St. Clair GK \& Woods B (1991) Explaining Change in Policy Subsystems: Analysis of Coalition Stability and Defection over Time. American Journal of Political Science 35: 851-880, Midwest Political Science Assoc. doi: $10.2307 / 2111497$
31. Heifetz RA (1994) Leadership without Easy Answers. The Belknap Press of Harvard University Press, Cambridge, MA.

32. Walker TC and Walker MK (2017) High reliability care and the challenges of opposing views: A policy and practice commentary. Maine Nurse Education Commission (MeNEC, April, 2017, state).

33. Wilson JQ (1989) Bureaucracy: What Government Agencies Do and Why They Do It. Basic Books.
Copyright: @2018 Mary K. Walker. This is an open-access article distributed under the terms of the Creative Commons Attribution License, which permits unrestricted use, distribution, and reproduction in any medium, provided the original author and source are credited. 